\title{
Lichtheimia corymbifera (Cohn) Vuill
}

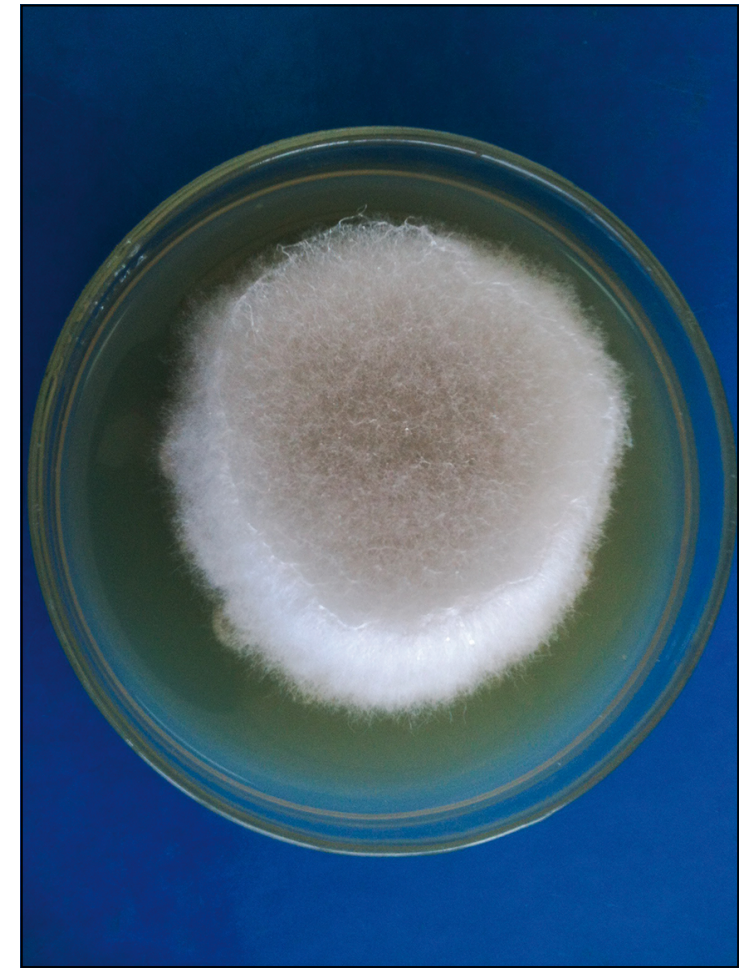

Figura 1. Colonia de Lichtheimia corymbifera en MEA a las 72 hrs de cultivo. Se observa el rápido crecimiento y la amplia ramificación.

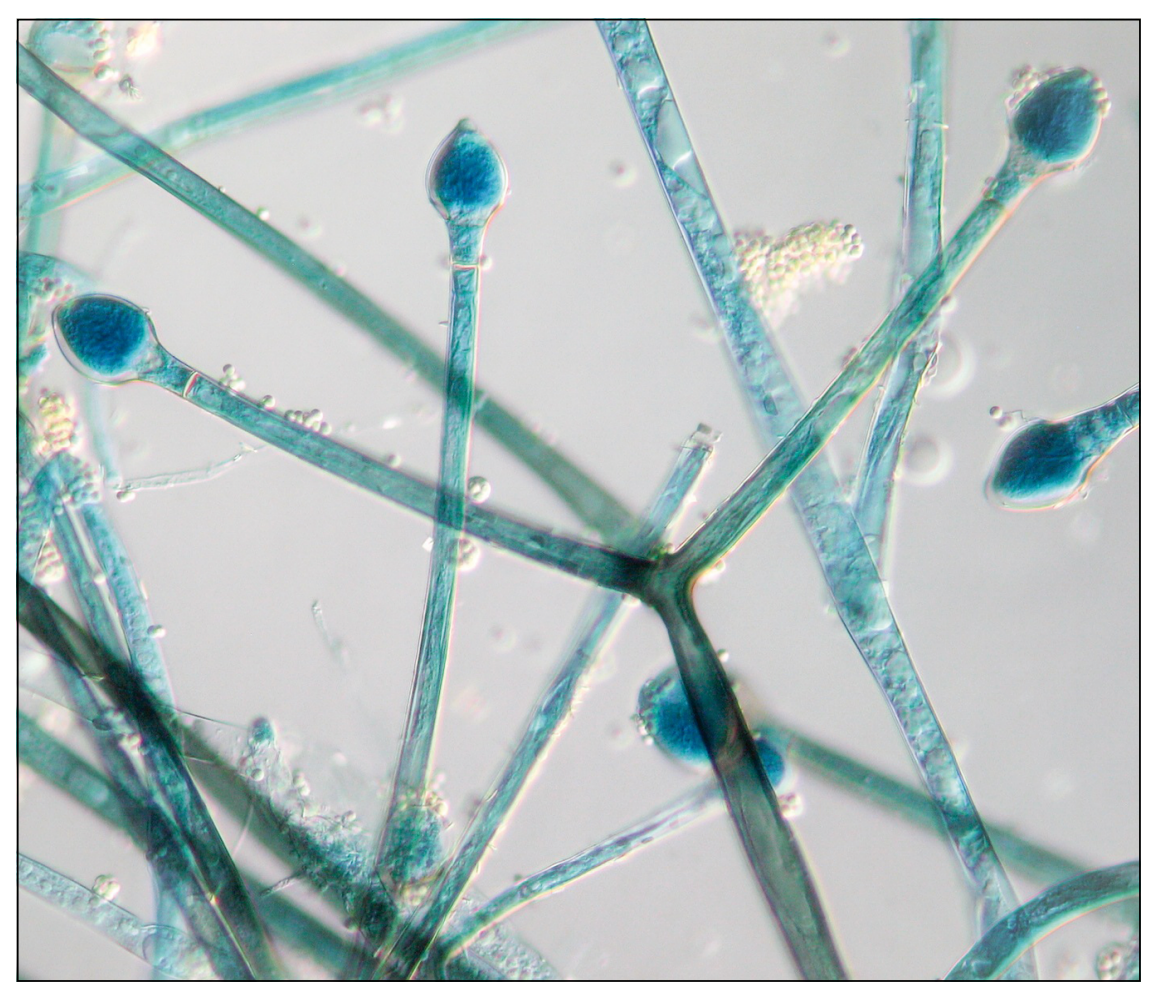

Figura 2. Micelio cenocítico con conidioforos de paredes lisas, columelas con apofisis cónica y septo debajo del esporangioforo. Tinción de lactofenol con azul de algodón. 100X. 


\title{
Lichtheimia corymbifera (Cohn) Vuill
}

Lichtheimia corymbifera es un hongo filamentoso inferior que pertenece al orden Mucorales, y que puede causar infección invasora pulmonar, rinocerebral, cutánea o diseminada en pacientes inmunocomprometidos. Está presente en una amplia variedad de sustratos, participando como otros hongos en la descomposición de la materia orgánica, por lo que juega un importante rol en el ciclo del carbono.

El género Lichtheimia está conformado por L. corymbifera, L. ornata, L. ramosa, L. hyalospora y L. sphareocystis. Las tres primeras especies tienen importancia clínica. Géneros afines como Absidia y Lentamyces no logran crecer a $37^{\circ}$ y $30^{\circ} \mathrm{C}$ respectivamente, por lo que en la actualidad no se les atribuye mayor importancia en micología médica.

Características macroscópicas: las colonias en agar con extracto de malta (MEA) a $30^{\circ} \mathrm{C}$ presentan un rápido crecimiento, son de color blanco a grisáceo, con un micelio ampliamente ramificado (Figura 1) y con lupa estereoscópica se observan estolones y rizoides. El diámetro de las colonias a $43^{\circ} \mathrm{C}$, a las $72 \mathrm{~h}$, es de aproximadamente $28 \mathrm{~mm}$ (tasa de crecimiento promedio de $0,4 \mathrm{~mm} / \mathrm{h}$ ); las esporas son elipsoidales a cilíndricas. Los esporangioforos generalmente se encuentran solitarios y nunca se disponen en forma opuesta a los rizoides, como en el género Rhizopus.

Características microscópicas: en muestras clínicas se pueden observar hifas cenocíticas gruesas, con ramificación en ángulo recto y en ocasiones, el esporangio, columela, o partes de éstos. En los cultivos, los esporangios son relativamente pequeños, piriformes, de paredes delicuescentes, de 100-120 $\mu \mathrm{m}$ de diámetro. La columela, que cubre 40-60\% del esporangio, es semiesférica, con una apófisis cónica característica; comúnmente se forma un septo único bajo el esporangio (Figura 2). Las esporas son esféricas o elipsoidales, de 3-4 $\mu \mathrm{m}$ de diámetro y de paredes lisas; las zigosporas son elipsoidales, entre suspensores, de 60-100 a 45-80 $\mu \mathrm{m}$ de diámetro.

Fisiología: No producen fermentación; son dependientes de tiamina y la máxima temperatura de crecimiento es de $48-53^{\circ} \mathrm{C}$.

Tratamiento: debe combinar el desbridamiento quirúrgico y antifúngicos sistémicos. Anfotericina $\mathrm{B}$ deoxicolato y sus formulaciones lipídicas tienen buena actividad sobre estas especies y se han usado tradicionalmente; posaconazol es una alternativa reciente. Lichtheimia corymbifera es resistente a fluconazol, voriconazol y equinocandinas.

\section{Referencias bibliográficas}

1.- Vuillemin P. Le genre Tieghemella et la série des Absidiées. Bull Soc Mycol Fr 1903; 19:119-27.

2.- Alastruey-Izquierdo A, Hoffmann K, de Hoog G S, Rodríguez-Tudela J L, Voigt K, Bibashi E, et al. Species recognition and clinical relevance of the zygomycetous genus Lichtheimia (syn. Absidia pro parte, Mycocladus). J Clin Microbiol 2010; 48: 2154-70.

3.- Alvarez E. El Subphylum Mucoromycotina: generalidades y aspectos taxonómicos recientes. Bol Micol 2013; 28: 16-25.

4.- Alastruey-Izquierdo A, Castelli M V, Cuesta I, Monzon A, Cuenca-Estrella M, Rodríguez-Tudela J L. Activity of posaconazole and other antifungal agents against mucorales strains identified by sequencing of internal transcribed spacers. Antimicrob Agents Chemother 2009; 53: $1686-9$.

\author{
Rodrigo Cruz \\ Laboratorio de Micología, Universidad de Valparaíso. \\ Karen Ducasse y Marcela González. \\ Hospital Gustavo Fricke, Viña del Mar. \\ Correspondencia a: \\ rcruzchoappa@gmail.com
}

